\title{
Anisotropy of strain hardening of the E110 alloy
}

\author{
P. V. Fedotov ${ }^{1}$, B. A. Kalin ${ }^{1}$, E. M. Morozov ${ }^{1}$, A. A. Mokrushin², \\ A. V. Kostyukhina ${ }^{\dagger, 3}$, R. N. Taktashev ${ }^{3}$ \\ †kostuhinaN@yandex.ru
}

\begin{abstract}
${ }^{1}$ National Research Nuclear University MEPhI, 31 Kashirskoe shosse, Moscow, 115409, Russia
${ }^{2}$ Luch Research Institute and Scientific Industrial Association, 24 Zheleznodorozhnaya St., Podolsk, 142100, Russia

${ }^{3}$ All-Russia Thermal Engineering Institute, 14 Avtozavodskaya St., Moscow, 115280, Russia
\end{abstract}

\begin{abstract}
The purpose of this work is to examine the deformation behavior and mechanical properties of the E110 alloy at room temperature, as well as to test the hypothesis about the isotropy of its strain hardening. The following specimens have been used in the experiments: ring specimens (tensile in the tangential direction), and segmented specimens (tensile in the axial direction). The tests have been carried out at room temperature on a universal testing machine that meets the requirements of State Standard Specification 28840-90, with a speed of a traverse moving of $1 \mathrm{~mm} / \mathrm{min}$. The processing of machine deformation diagrams was carried out in a standard way. As a result, the mechanical characteristics of the specimens made of E110 alloy in the axial and tangential directions are obtained. In addition, hardening curves of the E110 alloy for the axial and tangential directions in the coordinates $\ln (\sigma / 1 \mathrm{MPa})-\ln (e / 0.002)$ have been made. The investigations carried out made it possible to determine the presence of several sections on the deformation diagram (two for the axial direction, and three for the tangential) described by power functions and differing in the degree of hardening. It was also revealed that: the strain hardening of the E110 alloy is anisotropic; in the initial section of deformation, the strength of the alloy E110 in the tangential direction is higher than that is for the axial direction, in the final section it is lower; the error of approximation of experimental data in the case of using piecewise power functions is $7 \%$ for the axial direction, and $8 \%$ for the tangential direction; in the case of using power functions with a constant degree of hardening, the approximation error increases to $14 \%$, and $27 \%$ for the axial and tangential directions, respectively.
\end{abstract}

Keywords: fuel cladding, hardening curve, mechanical tests, alloy E110.

УДК: 620.172 .254

\section{Анизотропия деформационного упрочнения сплава Э110}

\author{
Федотов П. В. ${ }^{1}$, Калин Б. А. ${ }^{1}$, Морозов Е. М. ${ }^{1}$, Мокрушин А. А. ${ }^{2}$, \\ Костюхина А.В. ${ }^{\dagger, 3}$, Такташев Р. Н. ${ }^{3}$ \\ ${ }^{1}$ Национальный исследовательский ядерный университет «МИФИ», Каширское ш., 31, Москва, 115409, Россия \\ ${ }^{2}$ Научно-исследовательский институт научно-производственное объединение «ЛУЧ», \\ ул. Железнодорожная, 24, Подольск, 142100, Россия
}

${ }^{3}$ Всероссийский дважды ордена Трудового Красного Знамени Теплотехнический научно-исследовательский институт, ул. Автозаводская, 14, Москва, 115280, Россия

Целью настоящей работы является исследование деформационного поведения и механических свойств сплава Э110 при комнатной температуре, а также проверка гипотезы об изотропности его деформационного упрочнения. В экспериментах использовались: кольцевые образцы (растяжение в тангенциальном направлении) и сегментные образцы (растяжение в осевом направлении). Испытания были проведены при комнатной температуре на универсальной испытательной машине, соответствующей требованиям ГОСТ 28840-90, при скорости перемещения подвижного захвата 1 мм/мин. Обработка машинных диаграмм деформирования проводилась стандартным способом. В результате получены характеристики механических свойств образцов сплава Э110 в осевом и тангенциальном направлениях. Кроме того, были построены кривые упрочнения сплава Э110 для осевого и тангенциального направлений в координатах $\ln (\sigma / 1 \mathrm{MПa})-\ln (e / 0.002)$. Проведенные исследования позволили установить наличие на диаграмме деформирования нескольких участков (двух для осевого направления и трех для тангенциального), описываемых степенными функциями и отличающихся показателем степени упрочнения. Также было показано, что: деформационное упрочнение сплава Э110 анизотропно; на начальном участке 
деформирования прочность сплава Э110 в тангенциальном направлении выше таковой для осевого направления, на конечном участке - ниже; погрешность аппроксимации экспериментальных данных в случае использования кусочно-степенных функций составляет 7\% для осевого направления и $8 \%$ для тангенциального направления; в случае использования степенных функций с постоянной степенью упрочнения погрешность аппроксимации увеличивается до $14 \%$ и 27\% для осевого и тангенциального направления, соответственно.

Ключевые слова: оболочка твэла, кривая упрочнения, механические испытания, сплав Э110.

\section{1. Введение}

Работоспособность ядерного реактора при нормальной эксплуатации и в аварийных режимах во многом зависит от механических свойств конструкционных элементов его активной зоны. Твэл относится к первому (наиболее высокому) классу конструктивных элементов АЭС, влияющих на безопасность [5-7], что определяет высокий интерес к оболочечным материалам. Для изготовления оболочек твэлов реакторов на тепловых нейтронах используются сплавы циркония. В России для этих целей используется цирконий-ниобиевый сплав Э110, содержащий 0.95-1.05\% ниобия и легирующие добавки $(0.0006-0.012 \%$ железа и до $0.1 \%$ кислорода) [1]. Этот сплав обладает сочетанием таких свойств как малое сечение взаимодействия с тепловыми нейтронами, низкая активация при взаимодействии с нейтронами, прочность, коррозионная стойкость и др. [2-4].

В международной практике обоснования безопасности АЭС моделирование поведения твэлов в различных режимах эксплуатации выполняется расчетным путем с использованием вычислительных программ (расчетных кодов) [8-13]. Необходимой составляющей расчетных кодов являются модели деформирования оболочки твэла, записываемые, как правило, в виде функциональной зависимости напряжения течения от параметров деформирования (температуры, скорости деформации, величины пластической деформации и т.д.) [14-16]. Построение модели деформирования, кроме прочего, базируется на предположении об изотропном деформационном упрочнении материала, а также на предположении о монотонном характере зависимости напряжения течения от величины пластической деформации. Однако существуют данные о не монотонности деформационного упрочнения сплава Э110, а также о влиянии текстуры на его деформационное упрочнение [17-19].

В связи с чем, настоящая работа посвящена исследованию деформационного поведения сплава Э110 при комнатной температуре, а также проверке гипотезы об изотропности его деформационного упрочнения.

\section{2. Материалы и методы исследования}

Для исследования деформационного поведения сплава Э110 в осевом и тангенциальном направлениях были проведены испытания на растяжение сегментных и кольцевых образцов, изготовленных из одной оболочечной трубки твэла $\varnothing 9.13 \times 7.73$ мм.

Кольцевые образцы представляли собой отрезки оболочек высотой 2.7 мм с расчетной длиной рабочей части $L=6.3$ мм. Нагружение осуществлялось посредством полуцилиндрических опор диаметром 4 мм по схеме, представленной на Рис. 1. Подготовка образцов, a также проведение испытаний осуществлялись по методике [20]. Данная методика подразумевает предварительное распрямление образцов между опорами под нагрузкой, равной 95\% от предела текучести, определенного по методике [21], с последующим отжигом при температуре $550^{\circ} \mathrm{C}$ в течение двух часов. Такой подход позволяет исключить основную методическую погрешность, присущую испытаниям кольцевых образцов - не учёт деформаций при распрямлении [22].

Сегментный образец - отрезок оболочки твэла с двумя параллельными выфрезерованными рабочими частями длиной $L=6$ мм и шириной 3 мм, приваренный к резьбовым захватам, как показано на Рис. 2.

Необходимо отметить, что в отличие от проведенных ранее испытаний [23-26] в данной работе сначала были испытаны сегментные образцы, а затем из них же были изготовлены и испытаны кольцевые образцы. Кроме того, при обработке и анализе результатов испытаний внимание уделялось не только области больших пластических деформаций (>3\%), но и области малых деформаций.

Нагружение образцов осуществлялось при комнатной температуре с использованием универсальной испытательной машины, соответствующей ГОСТ 28840-90. Скорость перемещения подвижной опоры составила $1 \mathrm{мм} /$ мин.

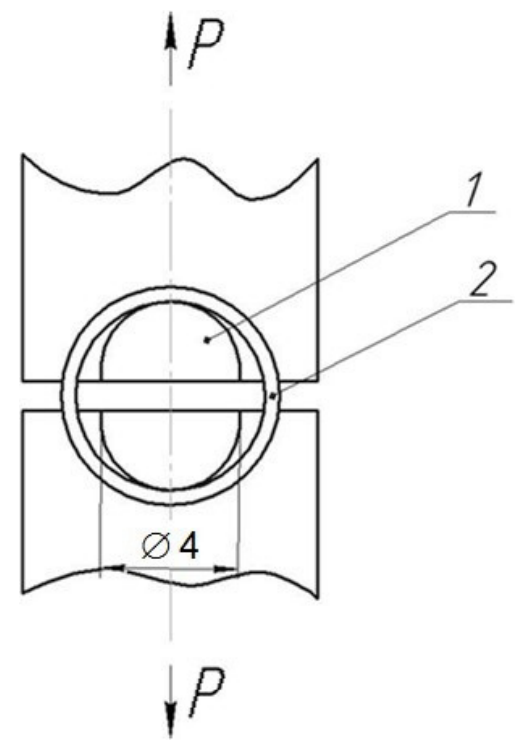

Pис. 1. Схема нагружения кольцевых образцов, 1 - опора, 2 - образец.

Fig. 1. Loading scheme for ring specimens, $1-$ mandrel, $2-$ specimen. 

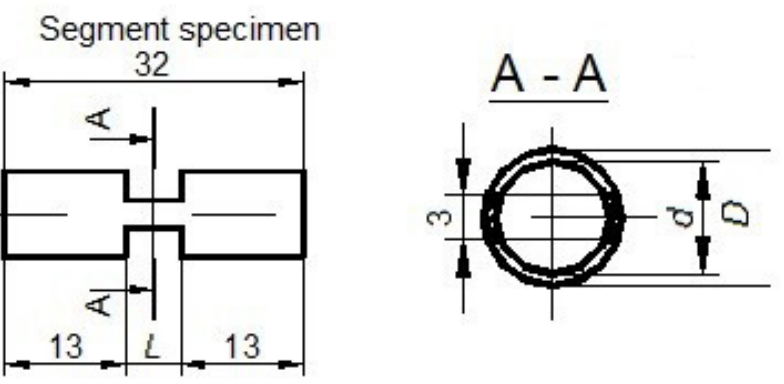

Рис. 2. Сегментный образец ( $d, D-$ внутренний и наружный диаметры оболочки).

Fig. 2. The segment specimen ( $d, D$ - inner and outer diameters of the cladding).

\section{3. Результаты и их обсуждение}

В результате механических испытаний по ГОСТ 28840-90 получены машинные диаграммы деформирования в координатах перемещение подвижной траверсы $(\Delta l)$ - сила $(P)$. Обработка машинных диаграмм деформирования проводилась стандартным способом в соответствии с методикой [27]. Для построения истинных диаграмм деформирования при деформациях, превышающих равномерное удлинение, часть образцов была испытана с промежуточными разгрузками и замерами текущих площадей поперечного сечения.

Экспериментальные данные о механических свойствах сплава Э110 в осевом и тангенциальном направлениях представлены в Табл. 1.

На Рис. 3 показаны истинные диаграммы деформирования (кривые упрочнения) в координатах $\ln (\sigma / 1 \mathrm{MPa})-\ln (e / 0.002)$ в осевом и в тангенциальном направлениях. Для их аппроксимации были выбраны кусочно-степенные функции, коэффициенты которых были получены методом наименьших квадратов [28]. Из приведенных данных следует, что на начальном участке деформирования прочность сплава Э110 в тангенциальном направлении выше таковой для осевого направления, при увеличении деформации картина изменяется.

Полученные результаты демонстрируют наличие на диаграмме деформирования нескольких участков, описываемых степенными функциями и отличающихся показателем степени упрочнения, а именно двух участков для осевого направления и трех - для тангенциального. При этом, кривые упрочнения сплава Э110 можно описать

Табл. 1. Механические свойства сплава Э110.

Table 1. The mechanical properties of E110 alloy.

\begin{tabular}{|c|c|c|}
\hline \multirow{2}{*}{$\begin{array}{c}\text { Свойство } \\
\text { Property }\end{array}$} & $\begin{array}{c}\text { Тангенциальное } \\
\text { Tangential }\end{array}$ & $\begin{array}{c}\text { Осевое } \\
\text { Axial }\end{array}$ \\
\hline $\begin{array}{c}\sigma_{0.2}, \mathrm{M} \text { На } \\
\text { YS, MPa }\end{array}$ & 301 & 236 \\
\hline $\begin{array}{c}\sigma_{\mathrm{B}}, \mathrm{M \Pi а} \\
\mathrm{UTS}, \mathrm{MPa}\end{array}$ & 358 & 397 \\
\hline $\begin{array}{c}\delta_{\mathrm{p}} \% \\
\mathrm{UE}, \%\end{array}$ & 11.8 & 16.9 \\
\hline
\end{tabular}

уравнениями (1), (2) для осевого и тангенциального направлений, соответственно:

$$
\begin{aligned}
& \sigma= \begin{cases}236 \cdot\left(\frac{\mathrm{e}}{0.002}\right)^{0.161}, & e<0.190 \\
129 \cdot\left(\frac{\mathrm{e}}{0.002}\right)^{0.294}, & e \geq 0.190\end{cases} \\
& \sigma= \begin{cases}301 \cdot\left(\frac{e}{0.002}\right)^{0.0499}, & e<0.0341 \\
242 \cdot\left(\frac{e}{0.002}\right)^{0.128}, & 0.0341 \leq e<0.201 \\
123 \cdot\left(\frac{e}{0.002}\right)^{0.274}, & e \geq 0.201\end{cases}
\end{aligned}
$$

С целью оценки погрешности аппроксимации для каждой $i$-той экспериментальной точки было вычислено значение величины $\left(\xi_{i}\right)$ по формуле:

$$
\xi_{i}=\frac{\sigma_{i}-\sigma\left(e_{i}\right)}{\sigma\left(e_{i}\right)},
$$

где $e_{i}-$ экспериментальное значение истинной деформации, $\sigma_{i}-$ экспериментальное значение напряжения течения, $\sigma\left(e_{i}\right)$ - значение напряжения течения для деформации $e_{i}$, вычисленное по формуле (1) для осевого направления или по формуле (2) для тангенциального направления.

Величина $\xi$ представляет собой случайную величину и является относительным отклонением экспериментальной точки от расчетной кривой.

Далее для осевого и тангенциального направлений было вычислено среднее арифметическое величины $\xi$ (выборочное среднее):

$$
\bar{\xi}=\frac{1}{N} \cdot \sum_{i=1}^{N} \xi_{i},
$$

а также оценка дисперсии этой величины (выборочная дисперсия):

$$
S^{2}=\frac{1}{N-1} \cdot \sum_{i=1}^{N}\left(\xi_{i}-\bar{\xi}\right)^{2}
$$

где $N$ - число экспериментальных точек. 


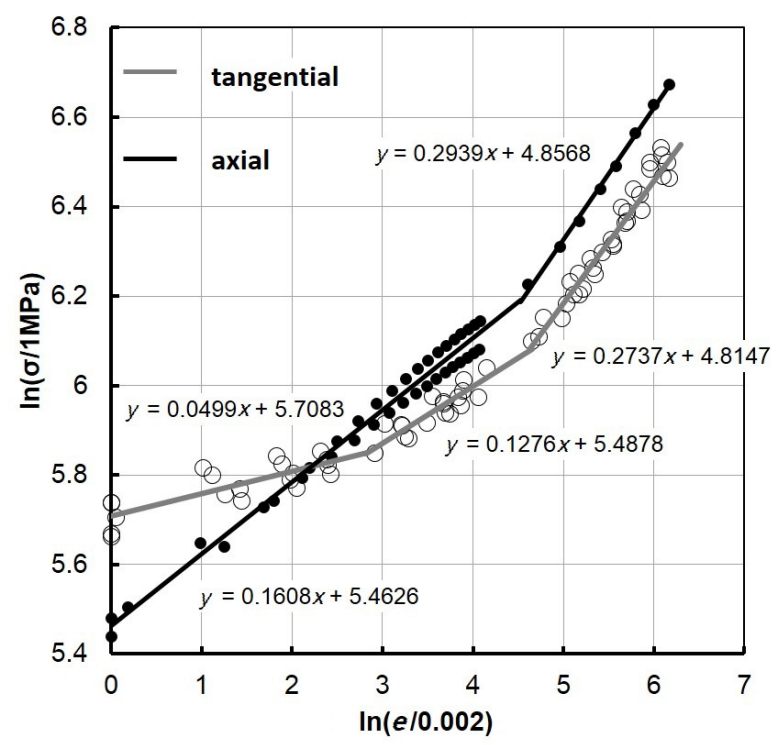

Рис. 3. Истинная диаграмма деформирования.

Fig. 3. True strain-stress diagram.

Следует отметить, что здесь и далее при статистической обработке используются термины и определения в соответствии с ГОСТ Р 50779.10-2000. Результаты вычислений приведены в Табл. 2. Так как в обоих случаях значение $\bar{\xi}$ более чем в пятьдесят раз меньше величины $S$, было сделано предположение, что $\bar{\xi}=0$. Проверка данной гипотезы осуществлялась по соотношению [29]:

$$
\frac{|\bar{\xi}|}{S / \sqrt{N}} \leq t_{\frac{\alpha}{2}},
$$

где $t_{\alpha / 2}-$ значение квантили статистики Стьюдента для числа степеней свободы $k=N-1$ и уровня значимости $a=0.05$. Сделанное предположение подтвердилось (Табл. 2).

Следует отметить, что эмпирическая функция распределения величины $\xi$, представленная на Рис. 4, практически совпадает с теоретической функцией нормального распределения для случайной величины $N\left(0, S_{2}\right)$.

В соответствии с ГОСТ Р ИСО 16269-6-2005 для величины $\xi$ были вычислены нижняя $(X L)$ и верхняя $(X U)$ границы статистического толерантного интервала 0.99/0.99 (доля совокупности, попадающая в границы данного интервала/уровень доверия):

$$
X_{L / U}= \pm k_{4}(N) \cdot S \text {, }
$$

где $k_{4}(N)-$ безразмерный коэффициент.

Результаты вычислений приведены в Табл. 2. Так как случайная величина $\xi$ является относительным отклонением экспериментальной точки от расчетной кривой и ее математическое ожидание равно нулю, границы ее статистического толерантного интервала могут быть интерпретированы как относительная погрешность аппроксимации, т.е. для тангенциального направления погрешность аппроксимации составляет 0.09 (9\%), а для осевого направления - 0.08 (8\%).

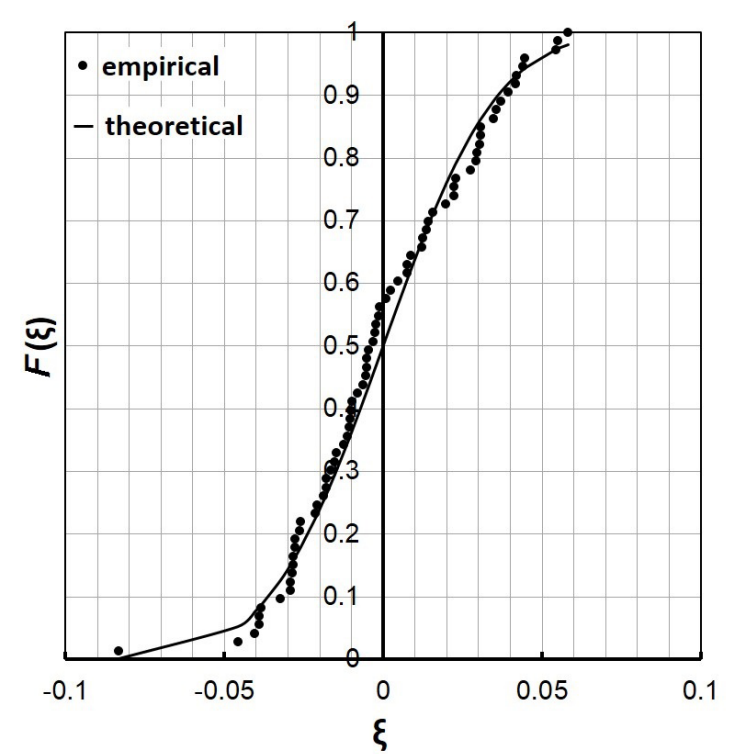

Рис. 4. Функция распределения (кольцевые образцы).

Fig. 4. Distribution function (ring samples).

Табл. 2. Числовые характеристики и анализ величины $\xi$.

\begin{tabular}{|c|c|c|}
\hline \multirow{2}{*}{$\begin{array}{c}\text { Числовые характеристики } \\
\text { Numerical characteristics }\end{array}$} & \multicolumn{2}{|c|}{ Направление / direction } \\
\hline & $\begin{array}{c}\text { Тангенциальное } \\
\text { Tangential }\end{array}$ & $\begin{array}{c}\text { Oсевое } \\
\text { Axial }\end{array}$ \\
\hline $\bar{\xi}$ & 0.000466 & 0.000449 \\
\hline$S$ & 0.0282 & 0.0229 \\
\hline$N$ & 73 & 45 \\
\hline$t_{\alpha / 2}$ & 2.3 & 2.3 \\
\hline$\frac{|\bar{\xi}|}{S / \sqrt{N}} \leq t_{\frac{\alpha}{2}}$ & 0.00193 & 0.00292 \\
\hline$k_{4}(N)$ & 3.23 & 3.45 \\
\hline$X_{L / U}$ & \pm 0.09 & \pm 0.08 \\
\hline
\end{tabular}
Table 2. Analysis of $\xi$ and its numerical characteristics.

\section{4. Заключение}

Настоящая работа посвящена исследованию деформационного поведения сплава Э110 при комнатной температуре, а также проверке гипотезы об изотропности его деформационного упрочнения. В результате проведенных исследований получено следующее:

1. На истинной диаграмме деформирования сплава Э110 в осевом направлении присутствует два участка степенного упрочнения с различными показателями степени упрочнения.

2. На истинной диаграмме деформирования сплава Э110 в тангенциальном направлении присутствует три участка степенного упрочнения с различными показателями степени упрочнения.

3. Кривые упрочнения пересекаются. При этом на начальном участке деформирования прочность сплава Э110 в тангенциальном направлении выше таковой для осевого направления, далее картина изменяется. 
4. Кривые упрочнения могут быть аппроксимированы кусочно-степенными функциями. Погрешность аппроксимации при этом составит для тангенциального направления 9\%, а для осевого направления - $8 \%$.

5. Таким образом подтверждено то, что деформационное упрочнение сплава Э110 не изотропно.

С другой стороны, как отмечалось во введении, для расчетов удобно пользоваться гипотезой об изотропности деформационного упрочнения. Ранее авторы работ [23-26] проводили проверку данной гипотезы на большом экспериментальном материале и продемонстрировали, что аппроксимация истинных диаграмм деформирования сплава Э110 монотонными степенными зависимостями со степенью упрочнения, независящей от направления испытания (и вообще от микроструктуры сплава) возможна при условии, что увеличение погрешности аппроксимации до 25 - 30\% не будет критичным.

\section{Литература/References}

1. A. V. Nikulina. Metal Science and Heat Treatment. 8, 7 (2003). (in Russian) [А. В. Никулина. МиТОМ. 8, 7 (2003).]

2. F.G. Reshetnikov, Yu.K. Bibilashvili, I.S. Golovnin. Razrabotka, proizvodstvo i ekspluataciya teplovydelyayushchih elementov energeticheskih reaktorov. Moscow, Energoatomizdat (1995) 320 p. (in Russian) [Ф.Г. Решетников, Ю.К. Бибилашвили, И.С. Головнин. Разработка, производство и эксплуатация тепловыделяющих элементов энергетических реакторов. Москва, Энергоатомиздат (1995) 320 c.]

3. A.S. Zajmovskij, A.V. Nikulina., N.G. Reshetnikov. Cirkonievye splavy v yadernoj energetike. Moscow, Energoatomizdat (1994) 256 p. (in Russian) [А. С. Займовский, А. В. Никулина, Н. Г. Решетников. Циркониевые сплавы в ядерной энергетике. Москва, Энергоатомиздат (1994) 256 c.]

4. E. Yu. Rivkin, B.S. Rodchenkov, V.M. Filatov. Prochnost> splavov cirkoniya. Moscow, Atomizdat (1974) 169 p. (in Russian) [Е. Ю. Ривкин, Б. С. Родченков, В.М.Филатов. Прочность сплавов циркония. Москва, Атомиздат (1974) 169 c.]

5. A.M. Bahmet'ev, O.B. Samojlov, G. B. Usynin. Metody ocenki i obespecheniya bezopasnosti YAEU. Moscow, Energoatomizdat (1988) 136 p. (in Russian) [А.м. Бахметьев, О.Б. Самойлов, Г.Б. Усынин. Методы оценки и обеспечения безопасности ЯЭУ. Москва, Энергоатомиздат (1988) 136 с.]

6. General Requirements of Safety Control of Nuclear Power Plants OPB-88/97 (PNAE G-01-011-97), 01.07.1998. (in Russian) [Общие положения обеспечения безопасности атомных станций. ОПБ-88/97 (ПНАЭ [-01-011-97), 01.07.1998.]

7. Nuclear Safety Regulations for Nuclear Power Plants. NP-082-07. Moscow, NTCYARB (2007) 48 p. (in Russian) [Правила ядерной безопасности реакторных установок атомных станций. НП-082-07. Москва, НТЦЯРБ (2007) 48 с.]
8. V.M. Troyanov, Yu. I. Lihachev, V.I. Folomeev. Izvestiya vuzov. YAdernaya energetika. 3, 19 (2002). (in Russian) [В.М. Троянов, Ю.И. Лихачев, В.И. Фоломеев. Известия вузов. Ядерная энергетика. 3, 19 (2002).]

9. G. Spykman, D. Martens, D. Bour. Proceedings of Enlarged Halden Programme Group Meeting. Sandefjord (2004) p. 62.

10. M.N. Jahingir, J. Alvis, R. O. Montgomery. Proceedings of the Water Reactor Fuel Performance Meeting. Kyoto (2005) p. 800.

11. J. Miettinen, J. O. Stengard, S. Kelppe. Proceedings of SEGFSM Topical Meeting on LOCA Issues Argonne National Laboratory. 2004 (CD-ROM).

12. P.V. Fedotov, A.A. Goncharov, A.V. Kumachev. Proceedings of International Atomic Energy Agency Technical Meeting. Ibaraki (2011) p. 279.

13. G. Khvostov, V. Novikov, A. Medvedev, S. Bogatyr. Proceedings of the Water Reactor Fuel Performance Meeting. Kyoto (2005) p. 992.

14. V. M. Troyanov, Yu. I. Lihachev, V.I. Folomeev. Izvestiya vuzov. Yadernaya energetika. 3, 14 (2002). (in Russian) [В.М. Троянов, Ю. И Лихачев, В.И. Фоломеев. Известия вузов. Ядерная энергетика. 3, 14 (2002).]

15. MATPRO - A Library of Materials Properties for LightWater-Reactor Accident Analysis. NUREG/CR-6150. V. 4. Rev. 2 INEL-96/0422.

16. A. Shestopalov, K. Lioutov, L. Yegorova. International Agreement Report. NUREG/IA-0209. NSI RRC KI 3067. IRSN-2002/33.

17. M. I. Alymov, G. N. Ermolaev, P. F. Prasolov. Collection of Proceedings of MEPhI. Moscow, Energoatomizdat (1985) p. 18. (in Russian) [М.И. Алымов, Г.Н. Ермолаев, П. Ф. Прасолов. В сборнике научных трудов МИФИ. Москва, Энергоатомиздат (1985) с. 18.]

18. P.F. Prasolov, V.P. Konoplenko, E.N. Pirogov, N.S. Surina, V.V. Novikov, V.I. Solyanyj. Collection of Proceedings of MEPhI. Moscow, Energoatomizdat (1982) p. 6. (in Russian) [П.Ф. Прасолов, В.П. Конопленко, Е.Н. Пирогов, Н.С. Сурина, В.В. Новиков, В.И. Соляный. В сборнике научных трудов МИФИ. Москва, Энергоатомиздат (1982) с. 6.]

19. A. B. Cohen, S. Majumdar, W.E. Ruther, M.C. Billone, H. M. Chung, L. A. Neimark. Summary of paper for 25th Water Reactor Safety Information Meeting. Maryland (1997) $19 \mathrm{p}$.

20. L.P. Loshmanov, P.V. Fedotov, A.V. Salatov, O. A. Nechaeva, N. V. Smirnov Proceedings of scientific session of MEPhI. Moscow (2007) p. 141. (in Russian) [Л.П. Лошманов, П.В. Федотов, А. В Салатов, О.А. Нечаева, Н. В. Смирнов. Труды Научной сессии МИФИ. Москва (2007) с. 141.]

21. Otraslevaya instrukciya OI 001.325-2006. (2006) 30 p. (in Russian) [Отраслевая инструкция ОИ 001.325-2006. (2006) 30 c.].

22. YA. B. Fridman. Mekhanicheskie svojstva metallov. Moscow, Gosudarstvennoe izdatel'stvo oboronnoj promyshlennosti (1952) 557 p. (in Russian) [Я.Б. Фридман Механические свойства металлов. Москва, Государственное издательство оборонной промышленности (1952) 557 с.] 
23. P. V. Fedotov, L. P. Loshmanov, A. V. Kostyukhina. Russian metallurgy (Metally). 9, 816 (2012). Crossref

24. P. V. Fedotov, L.P. Loshmanov, A.V. Kostyuhina, E. A. Olevskij. Izvestiya vuzov. Yadernaya energetika. 3, 164 (2012). (in Russian) [П. В. Федотов, Л. П. Лошманов, А.В. Костюхина, Е.А. Олевский. Известия высших учебных заведений. Ядерная энергетика. 3, 164 (2012).]

25. P. V.Fedotov, L. P.Loshmanov, A. V. Kostyuhina. Yadernaya fizika i inzhiniring. 3 (6), 507 (2012). (in Russian) [П.В. Федотов, Л.П. Лошманов, А.В. Костюхина. Ядерная физика и инжиниринг. 3 (6), 507 (2012).]

26. P. V. Fedotov, L.P. Loshmanov, A. V. Kostyuhina. Deformaciya i razrushenie materialov. 2, 29 (2013). (in Russian) [П.В. Федотов, Л.П. Лошманов,
А.В. Костюхина. Деформация и разрушение материалов. 2, 29 (2013).]

27. GOST 1497-84. Moscow. Standartinform (2005) 24 p. (in Russian) [ГОСТ 1497-84. Москва, Стандартинформ (2005) 24 c.]

28. N. Drejper, G. Smit. Prikladnoj regressionnyj analiz. Moscow. Finansy i statistika (1986) 369 p. (in Russian) [Н. Дрейпер, Г. Смит. Прикладной регрессионный анализ. Москва, Финансы и статистика (1986) 369 с.]

29. G. Korn, T. Korn, Spravochnik po matematike dlya nauchnyh rabotnikov i inzhenerov. Moscow. Nauka (1974) 832 p. (in Russian) [Г. Корн, Т. Корн. Справочник по математике для научных работников и инженеров. Москва, Наука (1974) 832 с.] 